\title{
NUMERICAL AND EXPERIMENTAL DESCRIPTION OF THE FLOW, BOUNDARY LAYER AND BED EVOLUTION IN BORE-DRIVEN SWASH ON A COARSE SEDIMENT BEACH
}

\author{
Riccardo Briganti', Nicholas Dodd ${ }^{1}$, Dubravka Pokrajac ${ }^{2}$ and Tom O’Donoghue ${ }^{2}$ \\ The paper presents the results of a comparison between a fully coupled numerical model for the hydro- and morpho- \\ dynamics of the swash zone. The model solves simultaneously the Non-Linear Shallow Water Equations and the \\ Exner equation for the bed updates. The model uses the simple Grass formula for the sediment transport and the \\ momentum integral method for the bottom shear stress prediction. The laboratory tests were carried out at the \\ University of Aberdeen swash facility and aimed at studying the hydrodynamics and sediment transport of a single, \\ bore-generated swash event. The comparison is carried out in terms of water depth and horizontal velocity (depth \\ average and profiles) and sediment transport. The model performs well in predicting these quantities, above all during \\ the run-up.
}

Keywords: swash-zone morphodynamics; coarse sediment beach; numerical modeling, TVD numerical schemes

\section{INTRODUCTION}

The accurate modelling of a number of coastal phenomena of engineering interest requires the simultaneous description of the flow and the bed evolution during bore-driven swash. Recent work (e.g. Briganti et al. 2011) has shown that the Non Linear Shallow Water Equations (NLSWEs), coupled with a simple bottom boundary layer model, are potentially able to provide a description of the flow structure in the water column. In particular, Briganti et al. (2011) compared the results of such a model with the experiments carried out in the swash facility at the University of Aberdeen on a fixed bed. The test consisted of generating a dam break and studying the subsequent flow on a 1:10 fixed slope. The comparison showed that the model is able to describe the evolution of the flow structure with a reasonably good accuracy, above all during the run-up stage. The bottom boundary layer model used, i.e. the simple momentum integral method, implies that the horizontal velocity in the boundary layer follows a logarithmic law. This has proven to be a good approximation also in field conditions (see Puleo et al, 2012).

The same experiments were then repeated using a mobile bed. This latter set of experiments will be considered here.

NLSWEs have been used in a number of studies to model a dam break on a mobile bed. The resulting flow and bed evolution have strong analogies with the flow during the run-up phase of a swash-driven event.

Briganti et al. (2012) developed a numerical model that can accurately predict the bed evolution driven by this type of flows using a simple and flexible numerical scheme aimed at being used for engineering applications. Recently Zhu et al. (2012), in a work aimed at achieving very high accuracy, showed that the model of Briganti et al (2012) (with the boundary layer description removed for the purposes of comparison) is also able accurately to describe the bed evolution during the swash event driven by a uniform bore on a mobile bed.

Here, this hydro- and morpho-dynamic model has been equipped with the boundary layer description included. The study shows the comparison of the simulated flow field and sediment transport with the measurements taken at the University of Aberdeen swash facility.

\section{NUMERICAL MODEL}

\section{Governing equations}

The proposed numerical model is based on the NLSWE coupled with the Exner equation for the bed evolution. Fig. 1 describes the variables of the problem along with the experimental set-up used in this study. The equations, in vector form, read:

$$
\partial_{t} W+\partial_{x} F(W)=S(W)
$$

Here $x$ is the abscissa and $t$ is time. $\boldsymbol{W}$ is the vectors of the unknowns, $\mathbf{F}$ is the fluxes vector and $\boldsymbol{S}$ is the vector of the source terms. These are defined as:

\footnotetext{
${ }^{1}$ Coastal Dynamics and Engineering Group,Faculty of Engineering, University of Nottingham, Nottingham NG7 2RD, U.K. Email: Riccardo.briganti@nottingham.ac.uk

${ }^{2}$ Department of Engineering, King's College, University of Aberdeen, Aberdeen AB24 3UE, U.K.
} 


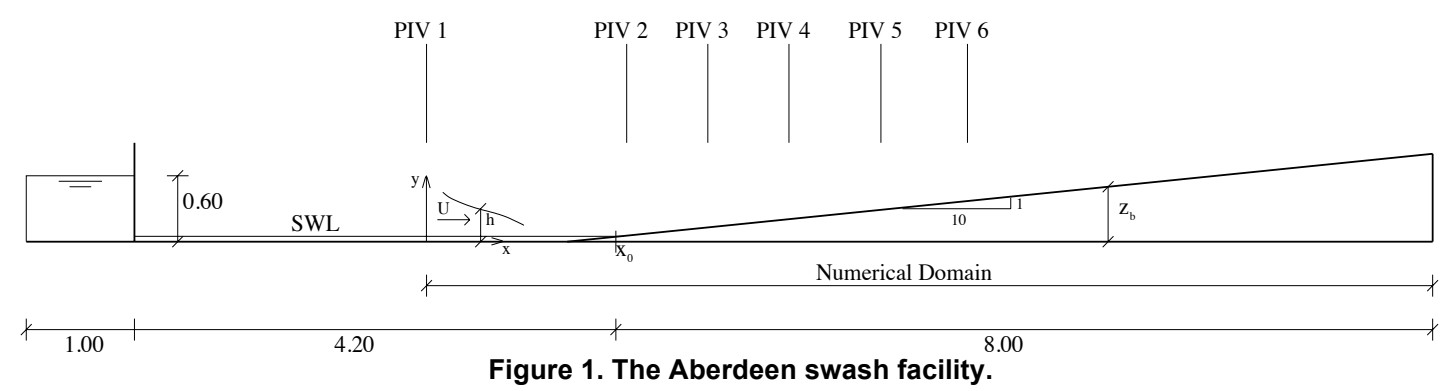

$$
W=\left[\begin{array}{c}
h \\
h U \\
z_{b}
\end{array}\right], \quad F=\left[\begin{array}{c}
g h \\
h U^{2}+\frac{1}{2} g h^{2} \\
\xi q_{s}
\end{array}\right], S=\left[\begin{array}{c}
0 \\
-g h z_{b, x}-\frac{\tau_{b}}{\rho} \\
0
\end{array}\right] \text { (2) }
$$

The unknown $U$ is the depth-averaged horizontal velocity, $h$ is the local water depth, and $z_{b}$ is the bed level. $\xi=1 /(1-v)$ where $v$ is the bed porosit. $q_{s}$ is the instantaneous sediment transport, $\tau_{\mathrm{b}}$ is bottom shear stress.

\section{Numerical Scheme}

The numerical scheme for the solution of the system has been presented in Briganti et al. (2012). Here only an outline will be given.

The system (1) is discretized in space into a set of $\mathrm{N}$ control volumes (cells of equal width $\Delta \mathrm{x})$.Each $\mathrm{i}^{\text {th }}$ cell $(i=1, N)$ has its centre located at $x_{i}$. We assume piecewise constant states in each cell. The system (1) is then solved using a Total Variation Diminishing (TVD) version of the classical MacCormack (MacCormack 1969) scheme.

This involves two stages (predictor and corrector):

$$
\begin{aligned}
& W_{i}^{p}=W_{i}^{n}-\frac{\Delta t}{\Delta x}\left(F_{i+1}^{n}-F_{i}^{n}\right)+\Delta t S_{i+\frac{1}{2}} \\
& W_{i}^{c}=W_{i}^{n}-\frac{\Delta t}{\Delta x}\left(F_{i+1}^{p}-F_{i}^{p}\right)+\Delta t S_{i+\frac{1}{2}}
\end{aligned}
$$

At a final stage the two stages the intermediate solutions are averaged and the scheme is equipped with TVD function $\boldsymbol{D}$ :

$$
W_{i}^{n+1}=\frac{1}{2}\left(W_{i}^{p}+W_{i}^{c}\right)+\frac{\Delta t}{\Delta x}\left(D_{i+\frac{1}{2}}-D_{i-\frac{1}{2}}\right)
$$

The function $\boldsymbol{D}$ requires knowledge of the eigenvectors and eigenvalues of the system (1), but, unlike Godunov-type schemes, no inter-cell Riemann problem is solved, resulting in a simpler and more flexible numerical scheme. Details about the function $\boldsymbol{D}$ are given in Briganti et al. (2012).

\section{Sediment transport formula}

The system (1) requires a sediment transport formula, i.e. a relationship between $q_{s}$ and the flow variables. Here the formula used is that Grass (1981) one, i.e.:

$$
q_{s}=A U^{3} .
$$


$A$ is the bed mobility parameter $\left(\mathrm{m}^{2} \mathrm{~s}^{-1}\right)$. This model has been tested with this formula in Briganti et al. (2012) for a dam break problem on mobile bed and in Zhu et al. (2012) for a swash problem.

\section{Bottom boundary layer model}

The momentum integral method (Fredsøe and Deigaard 1993) has been used in this study to model the bottom shear stress $\tau_{\mathrm{b}}$. The implementation of the method follows strictly Briganti et al. (2011) to which the reader is referred to for details.

The method postulates that the horizontal velocity profile in the bottom boundary layer is logarithmic and makes use of the dimensionless parameter $\mathrm{Z}$ defined as:

$$
Z=\frac{U_{0}}{U_{f}} \kappa,(6)
$$

where $U_{f}=\left(\tau_{\curvearrowleft} / \rho\right)^{s}$ is the friction velocity, and $U_{0}$ is the free stream velocity. $\kappa=0.41$ is the Von Karman constant. In order to compute $\tau_{\beta}$ from $U_{f}, \mathrm{Z}$ is computed by solving the ordinary differential equation:

$$
\frac{d Z}{d t}=\left[\frac{\kappa^{2} U_{0}}{z_{0}}-Z\left(e^{Z}-Z-1\right) \frac{1}{U_{0}} \frac{d U_{0}}{d t}\right] /\left[e^{Z}(Z-1)+1\right]
$$

which requires the knowledge of both $\mathrm{U}_{0}$ and its derivative in time. These quantities are computed using the knowledge of $U$ from the solution of the Non-Linear Shallow Water. Details can be found in Briganti et al. (2011). Note that here the thickness of the boundary layer is not limited to the water depth, i.e. $\delta$ can be such that the top of the boundary layer is located above the water column. This only means that only part of the logarithmic profile is developed in the flow and the free surface does not reach $U_{0}$. Also, the assumption of a logarithmic law for $\boldsymbol{U}$ implies that:

$$
Z=\ln \left(\frac{\delta+z_{0}}{z_{0}}\right)
$$

where $\delta$ is the bottom boundary layer thickness. $z_{0}$ is the height at which the horizontal velocity is assumed to be zero, $z_{0}=K_{n} / 30$ following Nikuradse (1932). Here $K_{n}$ is the bed roughness for which the Van Rijn (1982) model is used. Hence, $K_{n}=3 D_{50}$ where $D_{50}$ is the nominal diameter of the sediment.

Hence, by knowing $Z$ it is possible to estimate the bottom boundary layer thickness and, using the knowledge of $U_{0}$, the velocity profile.

\section{EXPERIMENTAL SETUP}

The experiments were carried out in the laboratory swash facility at the University of Aberdeen (U.K.). Kikkert et al. (2010) describe the setup and the experimental results in detail. The swash facility (Fig. 1) is built into a $20 \mathrm{~m}$ long, $0.9 \mathrm{~m}$ high and $0.4 \mathrm{~m}$ wide, glass-sided flume. The facility consists of a water reservoir placed inside the flume at one end. One of the reservoir walls is actually a gate, which is raised at high speed to produce the dam-break that generates the swash event on a beach with an initial slope of $1 / 10$. Two beaches were tested, a coarse sand one (target $D_{50}=15 \mathrm{~mm}$, measured $D_{50}=13$ $\mathrm{mm}$ ) and a gravel one (target $D_{50}=100 \mathrm{~mm}$, measured $D_{50}=84 \mathrm{~mm}$ ). Here only the coarse sand experiments will be considered. In the horizontal part in front of the reservoir, the flume has a fixed bed.

Measurements of the flow bed-parallel and bed-orthogonal velocity components and depth were taken along the beach in five PIV/LIF observation stations referred in Fig. 1 as PIV 2 to 6. Note that PIV 1 was not used in the mobile bed experiments but was used in the fixed bed experiment (see Kikkert et al. 2012 and Briganti et al. 2011).

The experiments were designed specifically to measure sediment transport during both the uprush and the backwash. To this end two different types of sediment traps, one for the uprush, one for the backwash, were used. In each case the experiments were repeated in order to allow measurements in multiple positions along the beach. These are coincident with the right (according to Fig. 1) 
boundary of PIV measurements areas from 3 to 5. Also, at each location the traps were released at different times during different repetitions of the experiments in order to obtain a time series of sediment transport rates during a swash event. These are shown in Fig. 6. Note that the release times were not equally spaced and the sediment discharge is considered constant between two samples.

\section{NUMERICAL TESTS}

Numerical tests setup

The numerical domain extends from the position of PIV 1 to the end of the beach. Boundary conditions are given at PIV 1. These are the same boundary conditions used in Briganti et al. (2011), i.e. the ensemble averaged measured time series of $h$ and $U$. Although no measurement of the flow at PIV 1 was made during the mobile bed experiments, the dam break and the resulting flow on the fixed horizontal bed were highly repeatable, giving confidence that the ensemble averaged $h$ and $U$ for the previous set of experiments can be used. Here $\Delta x=0.01 \mathrm{~m}$ and the minimum water depth used for the definition of the shoreline in the model is $h_{\min }=0.002 \mathrm{~m}$. Note that in the experiment the minimum water depth that could be measured was $h_{\min }=0.005 \mathrm{~m}$. This latter value is the one used for the comparisons of the position of the shoreline.

Eq. (5) has been calibrated using the maximum run-up point and the sediment transport measurements and the best fit has been obtained with $A=0.0004 \mathrm{~m}^{2} / \mathrm{s}$. As for the porosity, $v=0.5$.

\section{Results}

The numerical model results are compared with the measurements in terms of flow features and sediment transport.

Fig. 2 shows the comparison of the shoreline trajectories. The model overestimates the maximum run-up, a characteristic that was found also in Briganti et al. (2011), where a different scheme was used. Due to the steepness of the free surface at the tip during the run-up, the shoreline mismatch is minimum during this stage. However, during the backwash, as the swash lens stretches on the beach, this mismatch becomes more significant.

Despite the overestimation the time history of $h$ and $U$ at each measuring stations are well modelled, above all during the run-up phase as shown in Fig. 3.

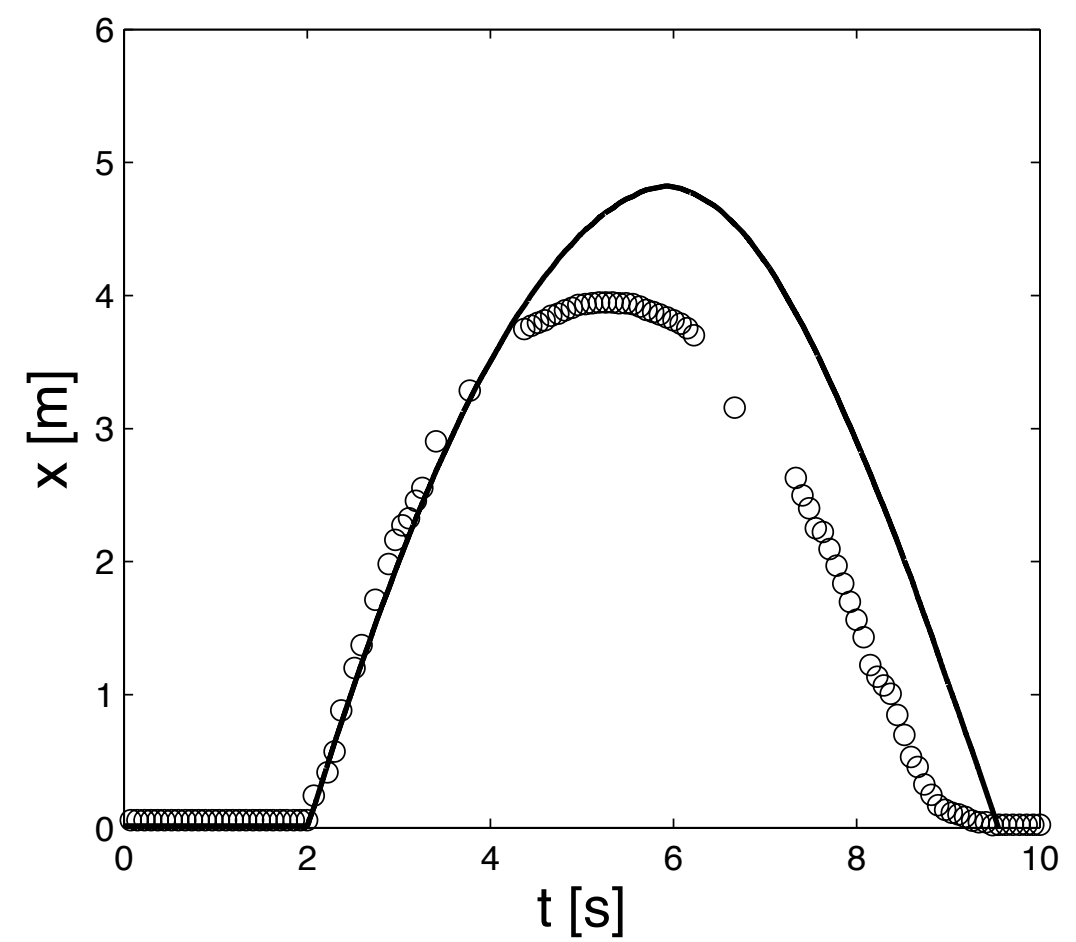

Figure 2. Numerical (solid black line) and experimental (black circles) shoreline trajectory.

The figure shows the comparison between the numerical and the experimental time histories of $h$ and $U$ along the beach. Three measurement stations are shown here, PIV 2 is located close to the 
initial position of the shoreline (thus in proximity of the bore collapse). PIV 3 and 5 are located further up the beach (see Fig. 1) and sediment transport measurements are also available for these two locations. The model performs very well in proximity of bore collapse in terms of $h$, while its performance deteriorates in the upper part of the beach, where the water depth is overestimated in each location.
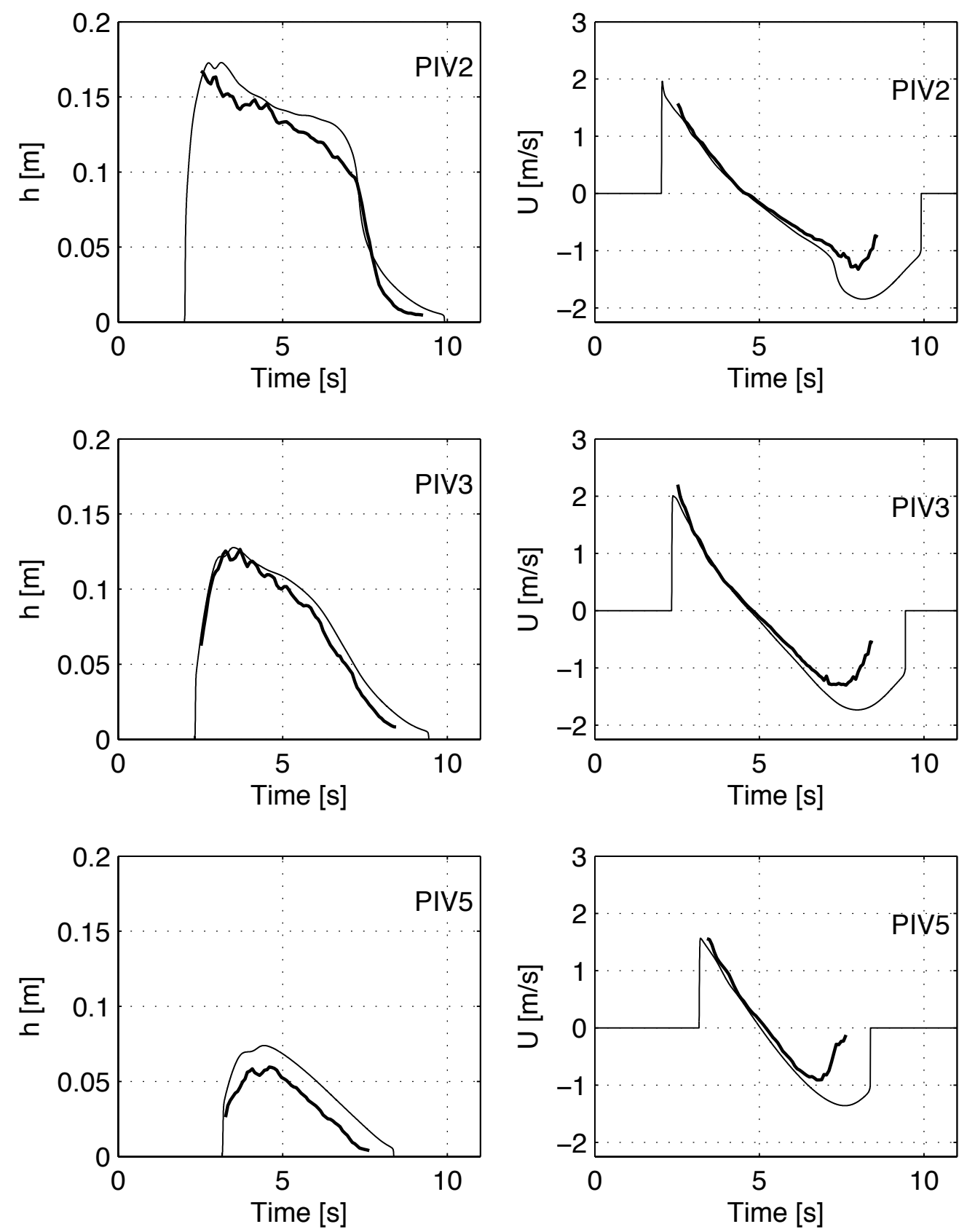

Figure 3. Numerical (thin black line) and experimental (thick black lines) water depth (left column) and depth averaged horizontal velocity (right column). 
Note that for the prediction of the sediment transport with Eq. (5) the accuracy in predicting $U$ is very important. The predicted flow velocity is in very good agreement with the data in the uprush. During the backwash is higher than the measured one. The flow, after reversal, reaches a minimum of the velocity ( $U$ is considered positive during run-up) before decelerating. The minimum of the velocity in the model is delayed with respect to the measurements. Also the predicted velocity magnitude is higher. Again, this behavior has been observed in the simulations of the flow on the fixed bed in Briganti et al. (2011).

The momentum integral method allows obtaining the horizontal velocity profiles together with the values of the bottom shear stress.

Fig. 4 shows the velocity profiles in two PIV measurement stations, PIV 3 and PIV 5, which are also coincident with the sediment traps. The agreement between the model and the measurements is fairly good, although the model seems to be in delay with respect to the experiments. In terms of bottom shear stress, some differences with the case of the fixed bed are noticeable. Fig. 5 shows the particles trajectories computed starting from the Eulerian flow field and the magnitude of shear stress (i.e. $\left|\tau_{b}\right|$ ) interpolated at the particles positions. The maximum $\left|\tau_{b}\right|$ is located above the still water level both in the uprush and the backwash. The larger differences between the fixed and mobile bed cases are at the end stage of the backwash, when the distribution of the stress among the different trajectories is different between the two cases. In the case of the mobile bed the stress is more evenly distributed among the trajectories. A comparison Fig. 17 of Briganti et al. (2011) also points out the difference in the trajectories between the two cases, given by the evolution of the bed during the backwash.

\section{Sediment transport}

The main purpose of the mobile bed experiments in the Aberdeen swash rig is the measurement of the sediment transport during the whole swash cycle. Thanks to the use of two different types of sediment traps it was possible to obtain the time history of the sediment transport rate $\left(q_{s}\right)$ both during run-up and backwash. Fig. 6 shows the experimental and numerical time history of the sediment transport at all three locations in which sediment traps were used (PIV 3, PIV 4 and PIV 5). The measured sediment transport rate is highest at the bore arrival, where the traps detected a peak. Subsequently, the flow transitions into a stage during which the sediment is at rest before the velocity of the flow during the backwash is large enough to mobilize the sediment. The numerical prediction agrees well with the sediment traps measurements, although some relevant differences are present. First, the predicted peak of the sediment transport is less sharp than the measurement, in all three locations. Also the model predicts sediment transport at the early stages of the backwash, while sediment traps do not measure any transport at this stage. It is not clear if this is a consequence of the Grass formula structure (i.e. lack of threshold) or because of the sensitivity of the traps. Further analyses with different transport formulas will be carried out. Consistent with the prediction of the velocity, the agreement with the data deteriorates in the backwash, where the sediment load is overestimated. However, the qualitative behaviour of $q_{s}$ is still well modelled. 

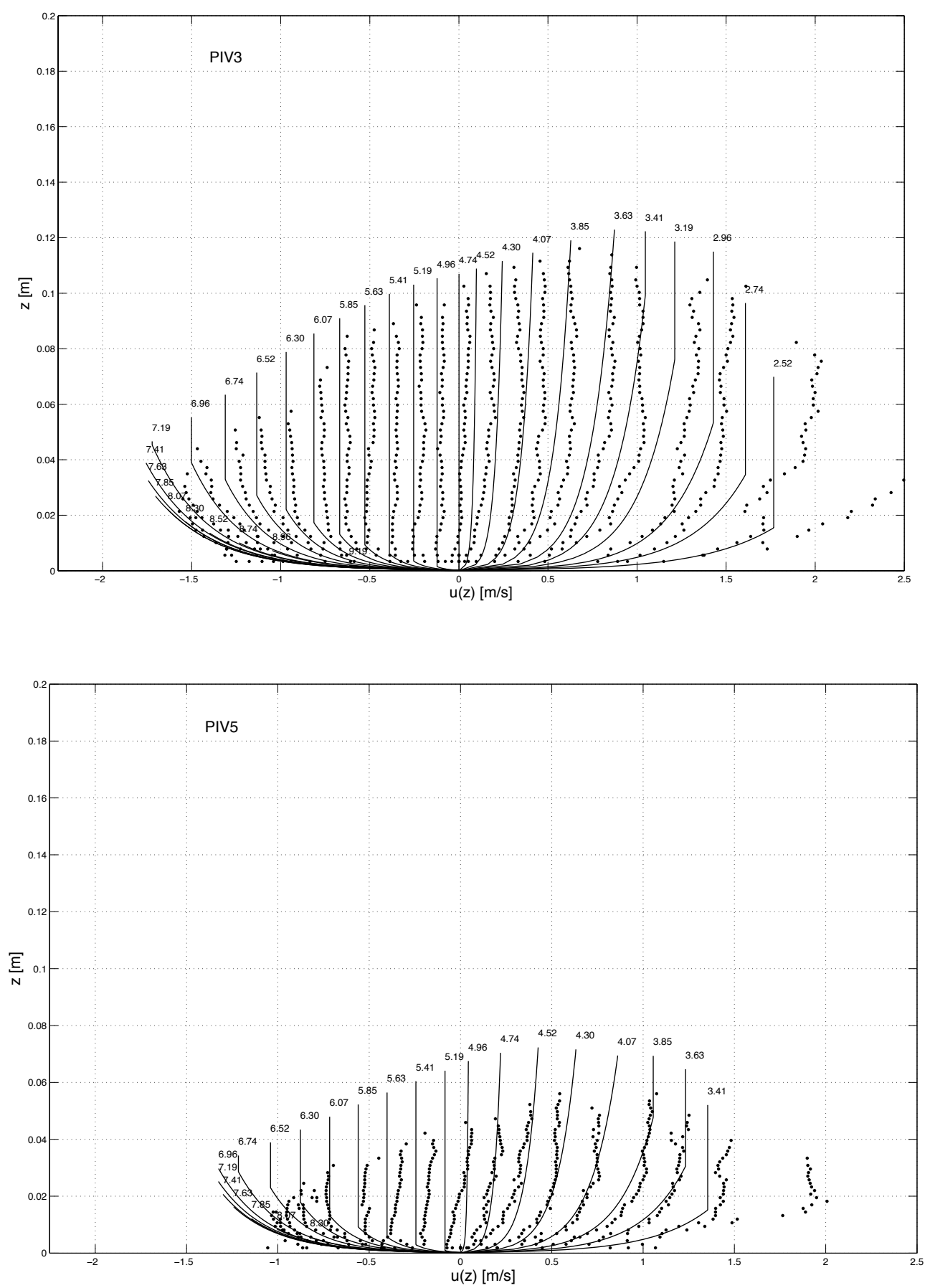

Figure 4. Numerical (black lines) and experimental (black dots) horizontal velocity profiles. 

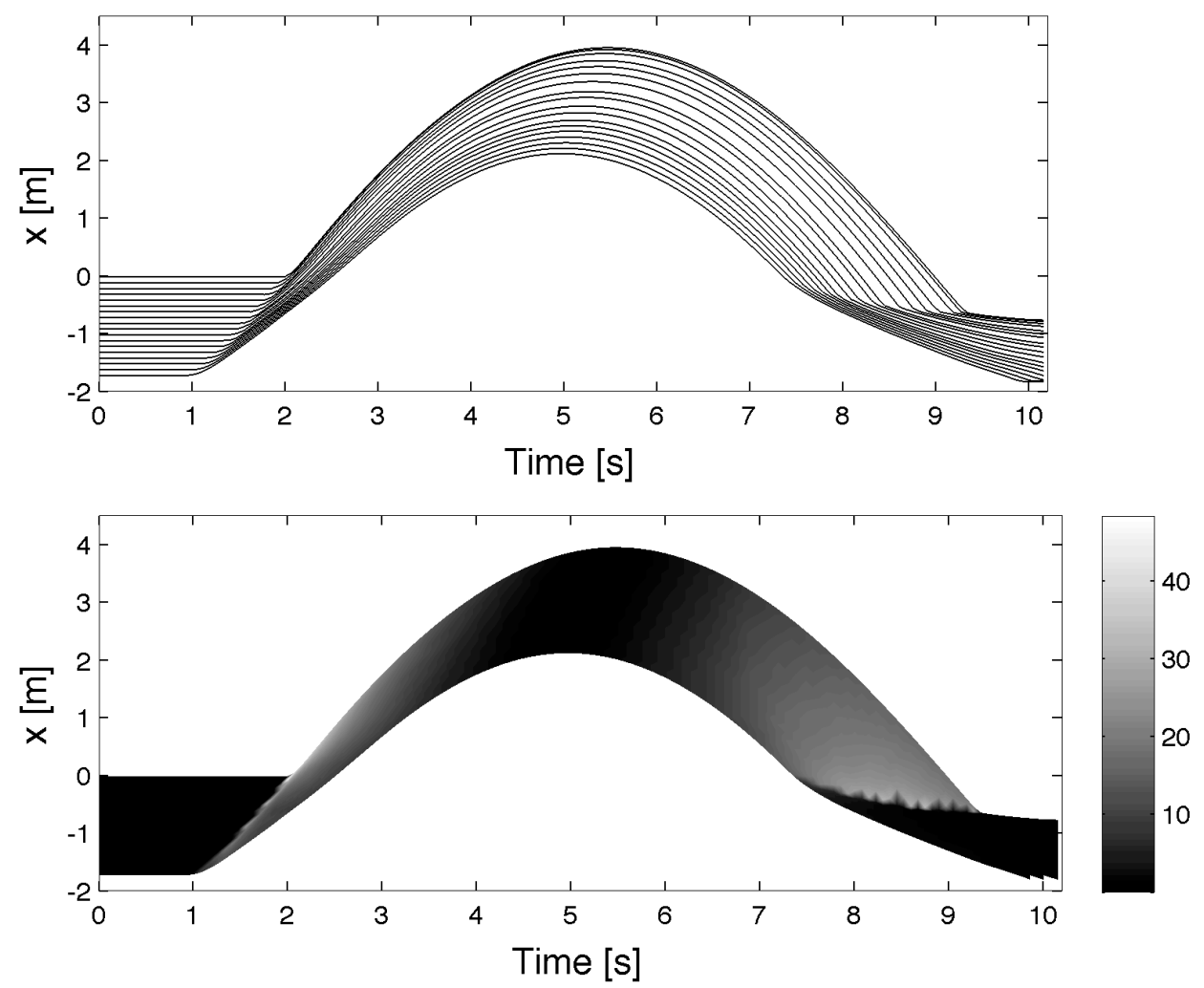

Figure 5. Upper Panel: computed particle trajectories. Lower panel: $\left|\mathrm{T}_{\mathrm{b}}\right|\left(\mathrm{Nm}^{-2}\right)$

\section{CONCLUSIONS}

A fully coupled model for the hydro- and morpho-dynamics of the swash zone has been tested against the laboratory tests carried out at the University of Aberdeen swash facility. The comparison shows that the model predicts fairly well the evolution of the flow field and the sediment transport in a single bore-generated, swash event.

The model uses the momentum integral method for the computation of the bottom boundary layer and the bottom shear stress. This method has been proven to perform well both in fixed bed laboratory tests (Briganti et al., 2011) and in field conditions (Puleo et al., 2012). Here it has been shown that it provides a fairly good quantitative prediction of the flow during the swash cycle.

As seen in the fixed bed experiments the model is more accurate in the run-up phase, while during the backwash the magnitude of the velocity is overestimated.

Similar considerations apply to the accuracy in the prediction of the sediment transport. The Grass model, which is widely used in research and practice, performs well in describing the sediment transport rate in the swash cycle.

The model, designed to be simple and computationally efficient in order to be used in engineering practice, has proven to provide a fairly accurate description of the flow and sediment transport during a single swash event on a coarse sediment beach. Future development will involve the comparison with sediment transport formulas with a threshold (such as the one proposed by MeyerPeter and Müller, 1948), and the inclusion of suspended sediment to allow the model to be able to predict the evolution of finer sediment beaches. 

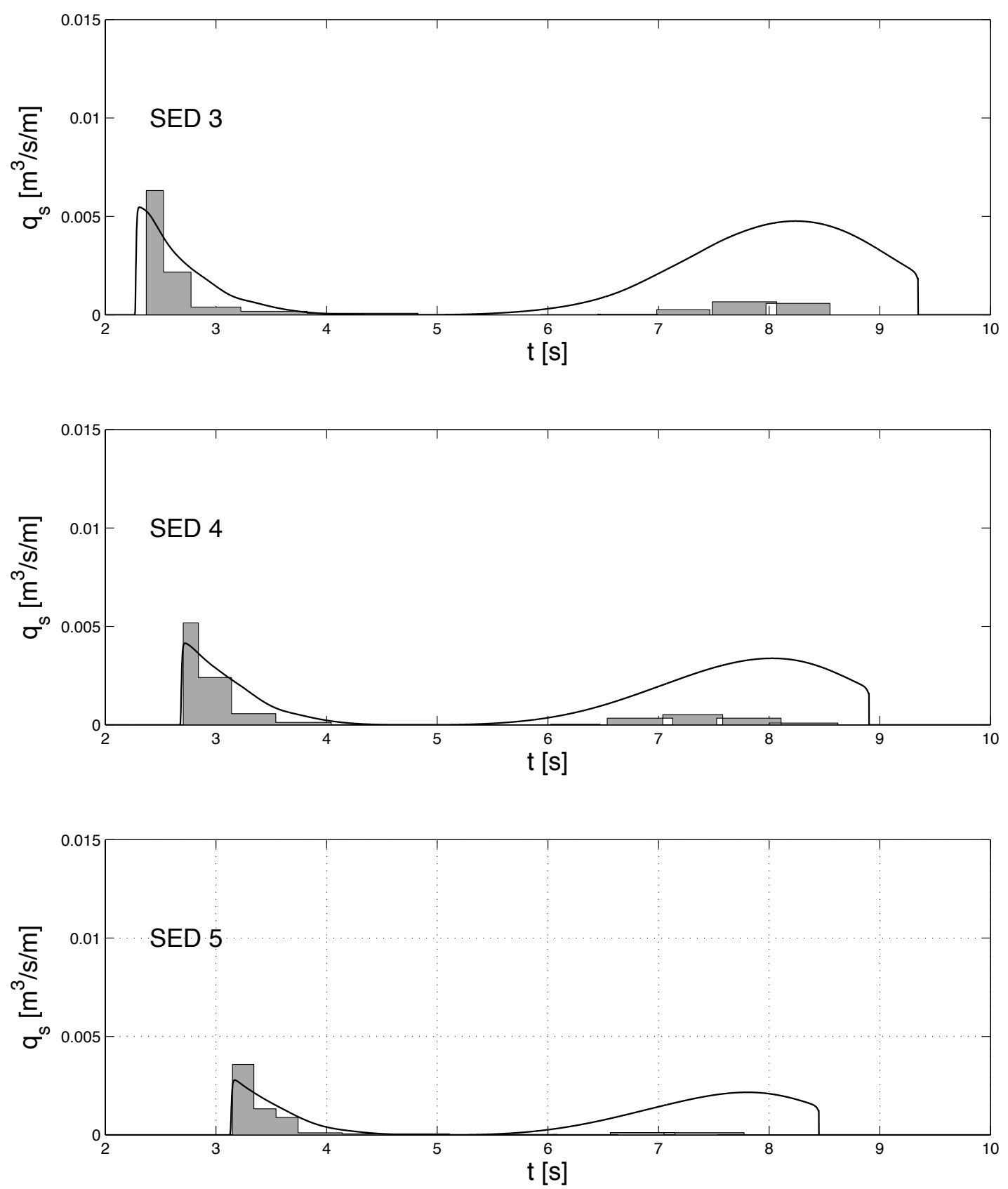

Figure 6. Upper Panel: Measured $q_{s}$ (gray bars) and computed $q_{s}$ (solid black line) at the sediment traps located at PIV 3. Lower Panel: Measured $q_{s}$ (gray bars) and computed $q_{s}$ (solid black line) at the sediment traps located at PIV 5.

\section{ACKNOWLEDGMENTS}

This study was supported by the EPSRC through the research project "Experimental and Numerical Modelling Study of Swash Zone Hydrodynamics and Sediment Transport" (EP/EO10407/1 and EP/E011330/1). Riccardo Briganti has been supported by the ESPRC Career Acceleration Fellowship $\mathrm{EP} / \mathrm{I004505/1}$. Their support is gratefully acknowledged.

\section{REFERENCES}

Battjes, J.A., and J.P.F.M. Janssen. 1978. Energy loss and set-up due to breaking of random waves, Proceedings of $14^{\text {th }}$ International Conference on Coastal Engineering, ASCE, 466-480.

De Vriend, H.J., J. Zyserman, J. Nicholson, J.A. Roelvink, P. Pechon, and H.N. Southgate. 1993. Medium-term 2DH coastal area modeling, Coastal Engineering, 21, 193-224. 
Wiegel, R.L. 1965. Oceanographical Engineering, Prentice-Hall, Englewood Cliffs, New Jersey, 531 $\mathrm{pp}$.

Briganti, R., N. Dodd, D. Pokrajac, and T. O'Donoghue. 2011. Non linear shallow water modelling of bore-driven swash: Description of the bottom boundary layer, Coastal Engineering, 58(6), 463477, doi:10.1016/j.coastaleng.2011.01.004.

Briganti, R., N. Dodd, D. Kelly and D. Pokrajac. 2012. An efficient and flexible solver for the simulation of the morphodynamics of fast evolving flows on coarse sediment beaches, Int. J. of Numerical Methods in Fluids, 69(4), 859-877, doi: 10.1002/fld.2618

Fredsøe, J., Deigaard, R., 1993. Mechanics of Coastal Sediment Transport. Vol. 3 of Advanced Series on Ocean Engineering. World Scientific, Singapore.

Kikkert, G.A., T. O'Donoghue, and D. Pokrajac. 2010. Laboratory measurements of hydrodynamics and sediment transport in the swash-zone, Environmental Hydraulics - Proceedings of the 6th International Symposium on Environmental Hydraulics, 1, 601-606.

Kikkert, G.A., T. O'Donoghue, D. Pokrajac and N. Dodd. 2012. Experimental study of bore-driven swash hydrodynamics on impermeable rough slopes, Coastal Engineering, 60, 149-166, doi:10.1016/j.coastaleng.2011.09.006.

Grass, A. 1981. Sediment transport by waves and currents. Report FL29, Ma. Tecnol., SERC London Cent.

MacCormack, R. 1969 The effect of viscosity in hypervelocity impact cratering. AIAA Hypervelocity Impact Conference, AIAA paper, 69-354.

Masselink, G., and J. A. Puleo. 2006. Swash-zone morphodynamics, Cont. Shelf Res., 26(5), 661-680, doi:10.1016/j.csr.2006.01.015

Meyer-Peter, E., and R. Müller 1948. Formulas for bed-load transport.Proc. 2nd Congress of the Int. Ass Hydraulic Structures Research, Stockholm.

Nikuradse, J., 1932. Gesetzmässigkeit der turbulenten Strömung in glatten Rohren. VDIForschungsheft.

Puleo, J. A., T. Lanckriet, P. Qang 2012 Near bed cross-shore velocity profiles, bed shear stress and friction on the foreshore of a microtidal beach Coastal Engineering, 68, 6-16,

Zhu, F., N. Dodd, and R. Briganti. 2012. Impact of a uniform bore on an erodible beach, Coastal Engineering, 60, 326-333, doi:10.1016/j.coastaleng.2011.08.006. 